\title{
A pesquisa em Nutrição Clínica no Brasil
}

\section{Research in Clinical Nutrition in Brazil}

Renata Borba de Amorim OLIVEIRA ${ }^{1,2}$

Shirley Donizete PRADO ${ }^{1}$

Maria Claudia da Veiga Soares CARVALHO ${ }^{1,3}$

Francisco Romão FERREIRA'

\section{R E S U M O}

Neste ensaio, busca-se uma primeira aproximação à pesquisa em Nutrição Clínica. Considera-se existir uma lacuna de informações e debates sobre elementos de cunho epistemológico e sobre interesses que marcam essa atividade no interior do campo científico da Alimentação e Nutrição no Brasil. O caráter multidimensional do campo alimentar-nutricional é apresentado como sua forte marca de identidade e motivo de distinção. Todavia, a pesquisa em Nutrição Clínica caracteriza-se pelo olhar biomédico, voltado enfaticamente para o metabolismo e para a doença, ficando excluídas as relações sociais e a intersubjetividade, com estudos voltados ao aspecto nutricional, sem considerar o alimentar. De outro lado, o alívio do sofrimento impõe a compreensão de relações entre seres humanos em sociedade. Tanto do ponto de vista metodológico como epistemológico, a biomedicina não é capaz de abarcar a totalidade do fenômeno da vida humana. Por essas razões, as Ciências Humanas e Sociais podem contribuir, em regime de cooperação interdisciplinar, com o campo da Alimentação e Nutrição, no sentido de instrumentalizar estudos inovadores tanto no aspecto conceitual quanto metodológico. Tais perspectivas podem trazer à luz as dimensões subjetivas do adoecimento humano, as quais constituem matéria essencial para os estudos sobre Nutrição Clínica.

Termos de Indexação: Alimentação. Ciências da Nutrição. Ciências Humanas. Dietoterapia. Pesquisa. Terapia nutricional.

\section{A B S T R A C T}

This essay intends to make a first approach to Clinical Nutrition research. We consider the gap that exists between information and debates about epistemological elements and about interests that mark this activity inside the scientific Field of Food and Nutrition in Brazil. The multidimensional character of the food-nutrition field is presented with its strong brand identity and motive of distinction. However, research in Clinical Nutrition

\footnotetext{
1 Universidade do Estado do Rio de Janeiro, Instituto de Nutrição, Programa de Pós-Graduação em Alimentação, Nutrição e Saúde. R. São Francisco Xavier, 524, Pavilhão João Lyra Filho, $12^{\circ}$ Andar, Bloco E, Sala 12.007, 20559-900, Rio de Janeiro, RJ, Brasil. Correspondência para/Correspondence to: SD PRADO. E-mail: <shirley.donizete.prado@gmail.com>.

2 Universidade Federal do Rio de Janeiro, Curso de Nutrição. Rio de Janeiro, RJ, Brasil.

${ }^{3}$ Universidade Federal do Rio de Janeiro, Instituto de Nutrição Josué de Castro, Programa de Pós-Graduação em Nutrição. Rio de Janeiro, RJ, Brasil.
} 
is characterized by the biomedical perspective that focuses emphatically on metabolism and disease, excluding the social relationships and intersubjectivity of the studies that centre on nutrition and ignore food. The relief from suffering requires understanding interpersonal relationships in society. Both from the methodological and epistemological viewpoints, biomedicine is not capable of encompassing the entirety of the human life phenomenon. For these reasons, Human and Social Sciences can provide an interdisciplinary contribution to the Field of Food and Nutrition, in the sense of instrumenting innovative studies conceptually and methodologically. Such perspectives can bring to light the subjective dimensions of human illness that correspond to the essential subject of study for Clinical Nutrition.

Indexing terms: Feeding. Nutritional sciences. Humanities. Diet therapy. Research. Nutrition therapy.

\section{N T R O D U Ç Ã O}

Neste ensaio, busca-se uma primeira aproximação à pesquisa em Nutrição Clínica. Considera-se existir uma lacuna de informações e debates sobre elementos de cunho epistemológico e sobre interesses que marcam essa atividade no interior do campo científico da Alimentação e Nutrição, da ciência e da sociedade no Brasil.

Esse esforço faz parte de um investimento mais amplo voltado para a problematização de relações sociais nos processos de definição de temáticas ${ }^{1}$ e de estabelecimento dos diversos núcleos de saberes², que compõem o campo científico da Alimentação e Nutrição ${ }^{3-5}$. Tal iniciativa vem sendo implementada dentro da perspectiva da afirmação do pluralismo de epistemologias como caminho de enriquecimento dos processos de construção de conhecimentos e saberes na lida acadêmica.

Considera-se, para as análises ora empreendidas, o aporte de Pierre Bourdieu às reflexões sobre campo científico ${ }^{6,7}$. Aqui, tanto os aspectos próprios da pesquisa (definição do objeto, procedimentos metodológicos e abordagens analíticas), quanto os agentes em relação no contexto da investigação científica, conferem o tom do debate. Desse modo, o mundo da ciência pode ser entendido como um "campo social como outro qualquer, com suas relações de força e monopólios, suas lutas e estratégias, seus interesses e lucros, mas onde todas essas invariantes revestem formas específicas" ${ }^{\prime 6}$ (p.122). Por esses caminhos, o campo científico pode ser considerado como um espaço de luta concorrencial pelo "monopólio da autoridade científica definida, de maneira inseparável, como capacidade técnica e poder social; ou, se quisermos, o monopólio da competência científica, compreendida enquanto capacidade de falar e agir legitimamente (isto é, de maneira autorizada e com autoridade), que é socialmente outorgada a um agente determinado" $^{6}$ (p.122).

O desenvolvimento teórico tomado de Thomas Khun ${ }^{8}$ também subsidia esta abordagem quando se têm em conta os movimentos expressos a partir de paradigmas que identificam distintas estruturas conceituais e as práticas delas decorrentes.

Assim, questões epistemológicas são tratadas como fenômenos sociais - como posicionamentos paradigmáticos, intrinsecamente políticos.

No que concerne aos aspectos epistemológicos, o campo da Alimentação e Nutrição corresponde a um espaço social em que as ordens da Natureza e da Sociedade se expressam através diferentes objetos de estudo ${ }^{4}$. O Quadro 1 sintetiza as especificidades que caracterizam cada uma dessas duas regiões da ciência: a Alimentação e a Nutrição.

O caráter multidimensional do campo alimentar-nutricional é apresentado por meio de discursos presentes em diversos tratados científicos $^{9-12}$ ou em documentos institucionais oficiais $^{13}$, através dos quais multi, inter ou transdisciplinaridade estão presentes como sua forte marca de identidade e motivo de distinção. Esta condição deveria, por conseguinte, implicar práticas pluriepistemológicas de valorização das caracte- 
Quadro 1. O campo científico da Alimentação e Nutrição.

\begin{tabular}{llll}
\hline Conceitos & & Domínio científico \\
\hline \multirow{4}{*}{ Alimentar } & $\begin{array}{l}\text { Comida } \\
\text { Trabalho, linguagem e simbolismo } \\
\text { Subjetividade. } \\
\text { Vida humana em sociedade. }\end{array}$ & $\begin{array}{l}\text { O campo da Alimentação trata da geração de saberes sobre } \\
\text { a comida, como mediadora de relações sociais entre seres } \\
\text { humanos. }\end{array}$ & Alimentação e \\
& Nutriente & O campo da Nutrição dirige-se para a produção de conheci- & Nutrição \\
Nutrir & Dieta. Clínica. Epidemiologia. & mentos sobre os nutrientes e suas interações nas células do & \\
& Biomedicina. & corpo humano normal ou patológico. & \\
& Vida biológica na natureza. & & \\
\hline
\end{tabular}

Fonte: Adaptado de Prado et al. ${ }^{4}$.

rísticas de origem de cada um desses espaços, como partícipes do jogo e da construção das regras de distribuição de capital científico em seu interior. Incluir-se-ia, nesse conjunto de práticas, a adoção de uma nomenclatura que viesse a representar, efetivamente, as variadas abordagens teóricas, os diferentes procedimentos metodológicos, os distintos agentes e as muitas forças que operam em seu interior, ou seja, um nome que corresponderia à expressão mais sintética de sua identidade, do seu "Ser": "Alimentação e Nutrição". Todavia, tal não se dá.

Muito mais que a mera escolha de palavras, expressa-se neste aparente não-movimento que mantém única a Nutrição, o movimento de não-visibilidade da Alimentação e, com ela, o silêncio retumbante sobre a subjetividade e sobre os sujeitos em seu viver desejante, esta que é a marca indelével da nossa Humanidade, justamente a que, em definitivo, distingue o homem dos demais seres vivos, animais ou plantas, e das máquinas.

\section{A nutrição clínica como um núcleo de saberes no interior do campo científico da alimentação e nutrição}

No caso brasileiro, estudos sobre o ordenamento interno ao campo da Alimentação e Nutrição informam sua conformação em espaços e formações disciplinares, ou seja, núcleos de saberes, como assim denomina Nunes ${ }^{14}$. Um largo predomínio da racionalidade biomédica dá o tom da maior parte desses núcleos de saberes no campo da Alimentação e Nutrição, registrando-se também a presença de perspectivas de cunho humanista, em que as relações sociais - e as subjetividades que nelas se estabelecem e se expressam -, são tidas em alta conta como desafios para a lida científica.

Não se trata de colocar essas duas abordagens como excludentes entre si. Há, sim, a predominância de núcleos de saberes que se caracterizam marcadamente pelo paradigma da biomedicina. Mas há, também, ainda que em proporções menores, espaços em que o olhar naturalizado se depara com questões de ordem política ou cultural ou filosófica, por exemplo, estabelecendo-se aí alguns diálogos profícuos. É um significativo alento e importante motivo de ânimo, perceber que referências como historicidade e reflexividade não estão de todo ausentes nos processos de produção de conhecimento no interior do campo científico em questão.

Em levantamento que trata das linhas de pesquisa que compunham os programas de pós-graduação em 2009 na área de avaliação denominada "Nutrição" na Coordenação de Aperfeiçoamento de Pessoal de Nível Superior (Capes), confirmam-se a diversidade e a riqueza epistemológicas como marca de identidade do campo científico alimentar-nutricional (Quadro 2) ${ }^{15}$. O núcleo de saberes que corresponde à Nutrição Clínica tem expressão nesse cenário, representado por $14,5 \%$ das linhas de pesquisa então em atividade nesse específico recorte da ciência nacional. 
Quadro 2. Linhas de pesquisa dos programas de pós-graduação distribuídos por núcleos de saberes que conformam o campo científico de Alimentação e Nutrição, em 2009, no Brasil.

\begin{tabular}{|c|c|c|}
\hline Núcleos de saberes & $\mathrm{N}^{*}$ & $\%$ \\
\hline 1. Alimentação e Nutrição em Saúde Coletiva & 29 & 42,0 \\
\hline $\begin{array}{l}\text { 1.1. Epidemiologia em Nutrição (Estudos epidemiológicos sobre nutrição e determinação do estado } \\
\text { nutricional em populações) }\end{array}$ & 14 & 20,3 \\
\hline $\begin{array}{l}\text { 1.2. Políticas de Alimentação e Nutrição (Estudos voltados para políticas, planejamento e gestão de pro- } \\
\text { gramas de alimentação e nutrição) }\end{array}$ & 9 & 13,0 \\
\hline $\begin{array}{l}\text { 1.3. Ciências Humanas e Sociais em Alimentação(Pesquisas qualitativas em alimentação e sociedade. } \\
\text { Estudos sobre cultura, educação, sociologia, epistemologia, comunicação, filosofia em alimentação) }\end{array}$ & 6 & 8,7 \\
\hline 2. Nutrição Básica, Experimental e Clínica & 24 & 34,8 \\
\hline $\begin{array}{l}\text { 2.1. Nutrição Básica e Experimental(Estudos bioquímicos, fisiológicos e genéticos sobre nutrição em ani- } \\
\text { mais de laboratório e humanos) }\end{array}$ & 14 & 20,3 \\
\hline 2.2. Nutrição Clínica(Estudos clínicos sobre nutrição de humanos) & 10 & 14,5 \\
\hline 3. Nutrição e Alimentos & 11 & 15,9 \\
\hline \multicolumn{3}{|l|}{ (Estudos sobre composição química, qualidade sanitária e tecnologia dos alimentos) } \\
\hline 4. Alimentação e Nutrição em Produção de Refeições & 5 & 7,2 \\
\hline \multicolumn{3}{|l|}{$\begin{array}{l}\text { (Estudos sobre produção e consumo de refeições em Unidades de Alimentação e Nutrição coletivas } \\
\text { e comerciais) }\end{array}$} \\
\hline Total & 69 & 100,0 \\
\hline
\end{tabular}

Nota: Há duplicidade nos registros acima, pois uma mesma linha de pesquisa, algumas vezes, contempla mais de um dos núcleos de saberes. Fonte: Adaptado de Kac et a/15.

Outro levantamento, bem recente, relativo a temas abordados em artigos publicados no periódico científico DEMETRA: Alimentação, Nutrição \& Saúde, entre 2000 e 2012, confirma esse perfil qualitativo em termos de núcleos de saberes que constituem o campo cientifico da Alimentação e Nutrição ${ }^{16}$. Registra-se a presença de artigos oriundos da Nutrição Clínica, representando cerca de $10 \%$ do total de publicações do periódico. Relevante salientar que essa é uma revista interdisciplinar e que recebe, portanto, contribuições de diversos espaços do conhecimento, com maior presença da área da Saúde e do campo alimentar-nutricional. Como revista nova, publica, principalmente, estudos desenvolvidos por pesquisadores brasileiros; tal condição a coloca em posição diferenciada em relação aos periódicos internacionais que gozam de imenso prestígio entre pesquisadores, universidades, instituições e agências de fomento à pesquisa e, por isso, mais procurada pelos estudiosos situados nos lugares biomédicos de produção de conhecimentos, em especial, os núcleos de saberes acima identificados como Nutrição Básica, Experimental e Clínica e Epidemiologia em Nutrição. Ainda que, portanto, não represente o universo dos estudos no campo da Alimentação e Nutrição no Brasil, esta sistematização deve ser aqui considerada, dado que corresponde a um dos poucos esforços já realizados a possibilitar algum vislumbre sobre a composição de forças sociais e de seus resultados materiais em termos de publicação científica.

Procedendo conforme estudos implementados $2,5,17$ a partir do Diretório dos Grupos de Pesquisa (DGP) ${ }^{18}$ do Conselho Nacional de Desenvolvimento Científico e Tecnológico (CNPq) e utilizando como termos de busca as expressões "nutrição clínica", "dietoterapia" e "terapia nutricional", recuperaram-se 39 grupos de pesquisa em atividade no Brasil no ano de 2010 (Figura 1). Complementarmente, consultando a Base Corrente desse mesmo Diretório, identificaram-se 88 grupos de pesquisa em agosto de 2014. Estudo implementado por Delmaschio et al. ${ }^{5}$ informa a existência de 472 grupos de pesquisa no campo da Alimentação e Nutrição no Brasil no ano de 


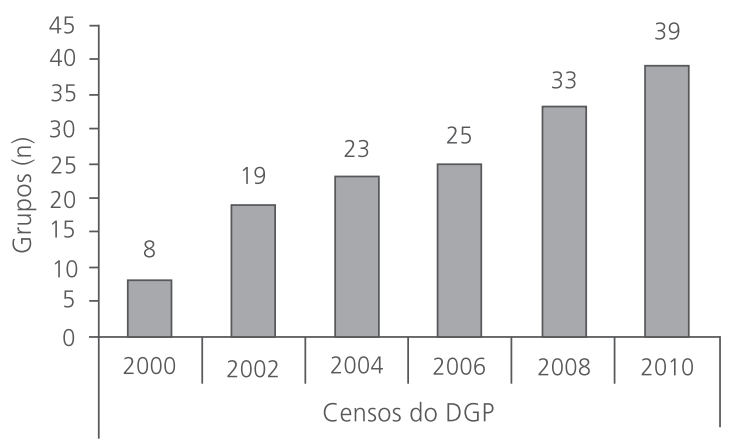

Figura 1. Grupos de pesquisa em Nutrição Clínica no Brasil de 2000 a 2010

Fonte: Conselho Nacional de Desenvolvimento Científico e Tecnológico ${ }^{18}$.

Nota: DGP: Diretório dos Grupos de Pesquisa.

2008; nesse momento, a pesquisa em Nutrição Clínica correspondia a $7 \%$ dos estudos situados no campo alimentar-nutricional. O conjunto de informações sugere que a Nutrição Clínica corresponde a algo entre $7 \%$ e $15 \%$ da pesquisa nacional quando esta se dirige aos estudos dos nutrientes e correspondentes necessidades celulares, assim como quando se volta para a compreensão dos sentidos e significados da comida em formações sociais específicas.

Numa abordagem de cunho qualitativo, chamam a atenção os nomes que identificam esses grupos de pesquisa.

Alguns são denominados por meio de expressões de ordem muito ampla, tomando a extensão do campo todo, como por exemplo: Alimentos, Nutrição ou Ciências Nutricionais, eventualmente especificando um grupo etário, como adultos ou idosos, por exemplo. Outros são intitulados de forma tão abrangente que praticamente tudo o que há de biomédico poderia caber em seu interior, como é o caso de Metabolismo ou Exercício Físico ou Comportamento Alimentar ou Coletividades Enfermas e Sadias. Há ainda os grupos que fazem uso de denominações correspondentes a doenças (como Cardiopatias, Câncer, Doenças Metabólicas, Diabetes) ou a especialidades médicas (Endocrinologia,
Gastroenterologia, Cirurgia) ou, ainda, disciplinas clássicas (Bioquímica, Fisiologia). Talvez seja um interesse implícito nessas denominações receber agentes de origens diversas, como um guarda-chuva. São ordenamentos institucionais que parecem agregar alguns pesquisadores ou docentes que desenvolvem ou pretendem desenvolver seus estudos, colocando lado a lado uma ampla gama de variados objetos e metodologias de pesquisa. Pode-se pensar que buscam abordagens plurais por meio da articulação entre distintas disciplinas em seu interior, o que corresponderia a um valor de distinção e expressaria ao menos alguma solidariedade entre os participantes da empreitada. Sem exclusão automática da possibilidade anterior, pode-se estar diante de uma significativa fragilidade de seus membros, ou de parte deles, que vão se aproximando e buscando algum fortalecimento conforme a vida institucional permite. Alguns tentam encontrar refúgio ou amparo entre pares que eventualmente os acolhem. Outros, usando vorazmente o espaço que se thes apresenta como mero degrau para o próximo passo na escalada de sua ascensão acadêmica. Isso, além de muitas tonalidades e matizes entre uma situação e outra.

Nesta aproximação primeira, percebe-se que a maioria dos grupos de pesquisa, em seus títulos, não apresenta palavras que possibilitem deduzir que ali se faz pesquisa em Nutrição Clínica. No interior desses grupos é comum encontrar uma única linha de pesquisa que guarda essa denominação ou algo similar, entre as tantas que os compõem; note-se que tais linhas de pesquisa são as que contam com o menor número de pesquisadores, muitas vezes, uma única pessoa e sem orientandos Esses arranjos no interior de grupos de pesquisa - que se caracterizam por uma mescla de diferentes abordagens e de objetos de estudo não necessariamente articulados entre si -, são bastante frequentes, estando os agentes menos capitalizados a participar de agregados científicos, que os recebem conforme seus próprios interesses, quando ainda não veem condições de seu estabelecimento específico. 
Um segundo conjunto desses grupos de pesquisa traz em seus nomes identificação que explicita diretamente a abordagem clínico-nutricional. Trazem, como nos casos anteriormente descritos, a presença de várias linhas de pesquisa, sendo que, desta feita, com maior número daquelas que se voltam para a Nutrição Clínica, e com maior número de pesquisadores e orientandos em sua composição. Mantêm-se, contudo, as expressões de ordem geral (como Metabolismo, Doenças Crônicas, Estresse Oxidativo ou disciplinas clássicas, como Fisiopatologia), conformando a indicação dos estudos aí empreendidos.

Contando com significativo número de nutricionistas, esses espaços de convivência com outros profissionais correspondem a lugares em que tais agentes podem expressar suas forças em crescimento. Se, entre 2000 e 2010, o número de grupos de pesquisa cuja área predominante era a medicina passou de 3 para 5, para a área predominante Nutrição esse número passou de 5 para 30; registre-se que, ao lado do intenso incremento na Nutrição, não há crescimento expressivo da pesquisa em Nutrição Clínica em outras áreas - a como Enfermagem, Farmácia, Saúde Coletiva, por exemplo -, ainda que se deva notar sua existência (Figura 2).

Nos dias de hoje, valor e distinção são conferidos à produção científica de rápido consumo em periódicos internacionais presentes em duas principais bases de indexação (Journal Citation Reports ${ }^{19}$ e SCImago Journal $/ s^{20}$ ), modelo em que predominam as Ciências da Natureza. Construir esse tipo de conhecimento e alcançar sua divulgação em conformidade com os referenciais normativos vigentes são os caminhos para a capitalização nos meios científicos, e o Brasil não foge a esse padrão. Contudo, aqui também não há homogeneidade. Estudos clínicos e biomédicos não mantêm similaridade permanente entre si, e alguns gargalos são particularmente identificados entre os primeiros. A pesquisa clínica caracteriza-se por demandar tempo muitas vezes bastante prolongado; as questões éticas são particular-

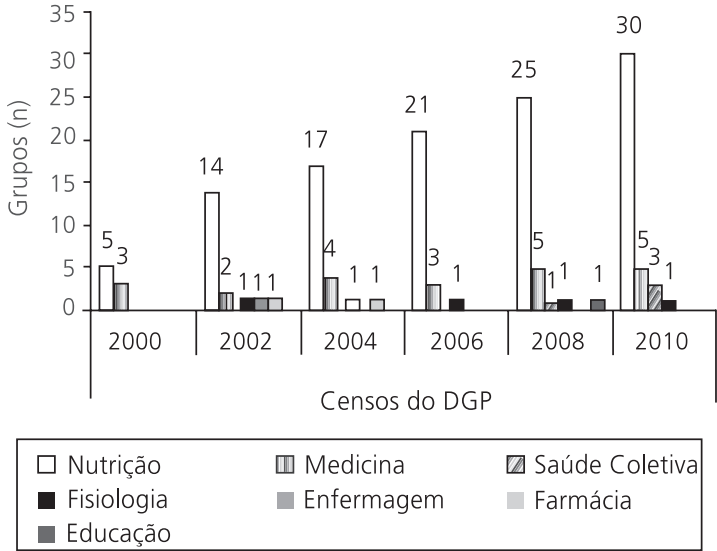

Figura 2. Grupos de pesquisa em Nutrição Clínica segundo Área Predominante no Brasil, de 2000 a 2010.

Fonte: Conselho Nacional de Desenvolvimento Científico e Tecnológico ${ }^{18}$.

Nota: DGP: Diretório dos Grupos de Pesquisa.

mente complexas; o acompanhamento dos pacientes exige controles, em muitos casos, de difícil execução; é limitada a rede de instituições que apresentam condições para o desenvolvimento dessas investigações; há dificuldades na construção de bases de dados que permitam usos por diversos pesquisadores; a dependência da disponibilidade de voluntários é um importante fator de limitação; é baixo o financiamento para essa atividade quando comparado a outros espaços da pesquisa biomédica ${ }^{21,22}$.

Diante desses entraves, é comum a migração de pesquisadores da pesquisa clínica, incluídos os que atuam na Nutrição Clínica, para campos próximos nos quais há maior viabilidade para o desenvolvimento de suas atividades. Passam, assim, a se dirigir a outros objetos como aqueles situados no âmbito da Bioquímica, da Fisiopatologia, da Genética, da Epidemiologia... . Enfim, ao se verem mergulhados no agressivo modelo competitivo da ciência atual, os agentes (pesquisadores, estudantes, grupos de pesquisa, instituições etc.) seguem participando e construindo o jogo científico e suas regras, e construindo a si mesmos nesse complexo processo. 
Debaixo do guarda-chuva das denominações biomédicas mais amplas ou apresentando-se de modo mais específico, a pesquisa em Nutrição Clínica vem crescendo, ao mesmo tempo em que se vê constantemente diante do importante dilema: permanecer nadando contra a corrente e/ou migrar para alguma facilidade em outros campos?

Parece razoável, aqui, pensar na existência de multi ou interdisciplinaridade entre a pesquisa clínica e os demais estudos biomédicos como caminho indiscutível para o núcleo de saberes da Nutrição Clínica. Todavia, é imprescindível registrar que a clínica refere-se a seres humanos em relação. A clínica para seres humanos exige alguém em sofrimento e outro alguém cuja atividade corresponde à busca de caminhos para o alívio desse sofrer. São pessoas que se relacionam entre si e com instituições, empresas, governos, enfim com ordenamentos sociais de todo tipo, expressando forças e interesses os mais diversos.

Nesse processo, destacam-se os interesses de cunho mercadológico, como, por exemplo, aqueles que se expressam através da chamada medicalização da sociedade ${ }^{23}$, que vem dando o tom dos (des)encontros no âmbito da saúde, da prática clínica e, também, da pesquisa clínica, incluída aí a de caráter nutricional. Desde fenômenos macrossociais até o mais íntimo da relação entre o profissional de saúde e seu paciente - passando pelas mediações situadas nas esferas da política, da cultura, dos valores que circulam nas formações sociais -, há toda a subjetividade inexoravelmente presente, e que a pesquisa clínica, incluindo a que se dá no âmbito da Nutrição Clínica, parece ignorar.

A nuvem de palavras (Figura 3) construída a partir dos nomes das linhas de pesquisa que compõem os grupos de pesquisa aqui trazidos para exame coloca, de forma cristalina, a predominância da racionalidade biomédica no âmbito nutricional e a ausência dos sujeitos implicados nesses estudos. Na imagem gerada pela construção da nuvem, destaca-se uma terapêutica voltada enfaticamente para o Metabolismo e, com menor força, para a doença. A palavra paciente aparece uma única vez, sendo um verdadeiro desafio encontrá-la na Figura 3.

O paciente se configura como um sujeito assujeitado, colocado na condição de objeto de

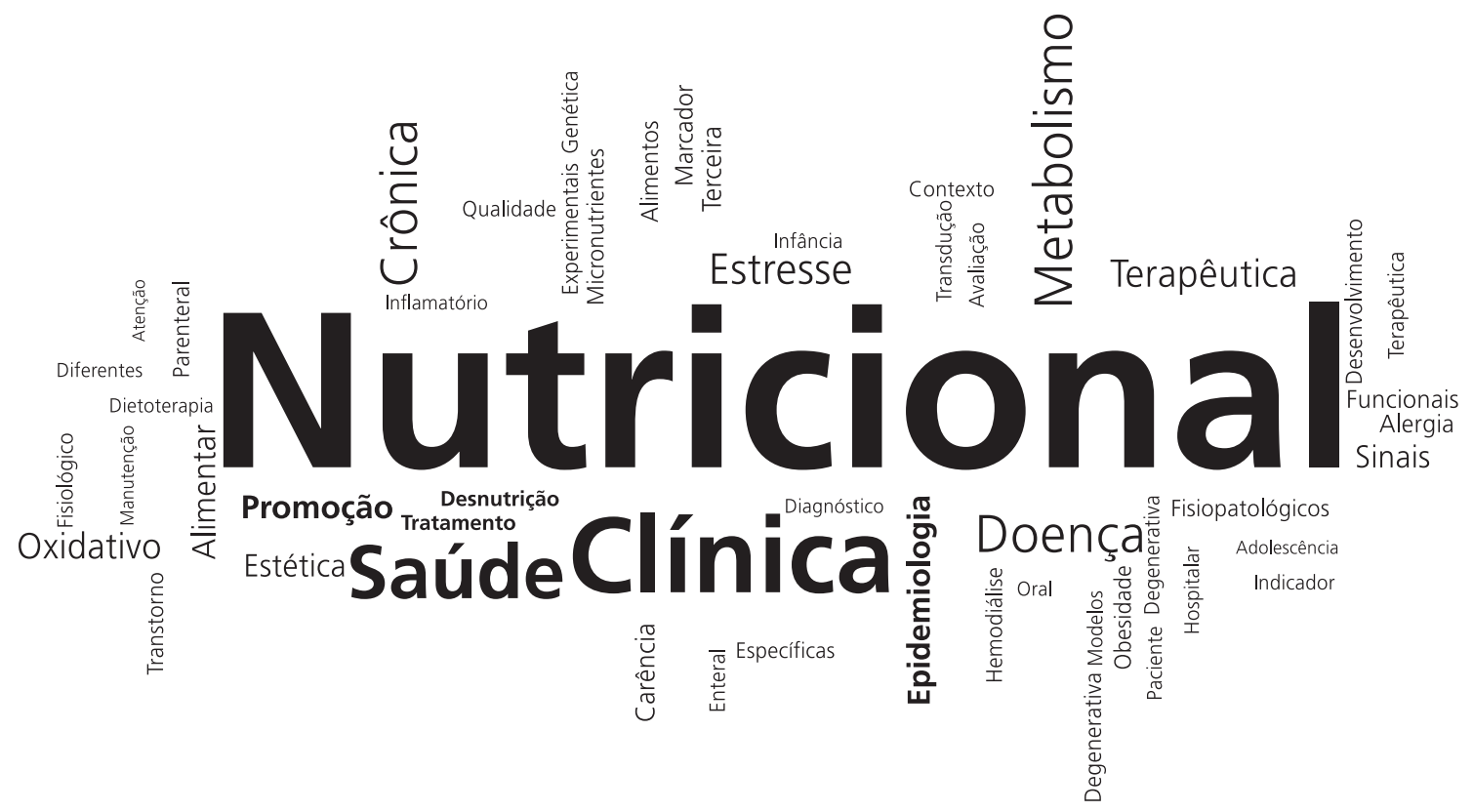

Figura 3. Nuvem de palavras dos grupos de pesquisa cujo nome explicita sua abordagem clínico-nutricional, em 2014, no Brasil. 
uma terapêutica definida por outrem. Mesmo esse outro, que domina a cena diante do paciente, não aparece. Assim, excluem-se as relações sociais e a intersubjetividade nos estudos desenvolvidos neste recorte da pesquisa, que se fixa no aspecto nutricional, sem considerar o alimentar. O alívio do sofrimento, imposto pelas relações entre seres humanos em sociedade, fica então reduzido a um olhar que ignora o relacional, perscrutando apenas o corpo biológico.

\section{Pesquisa em nutrição clínica e perspectivas interdisciplinares no campo da alimentação e nutrição}

Em praticamente todas as culturas, os alimentos sempre foram relacionados com a saúde - não apenas porque sua abundância ou escassez colocam em questão a sobrevivência humana, mas também (e em particular no mundo ocidental contemporâneo) porque o tipo de dieta e as razões médicas para sua utilização influenciam fortemente a atitude diante da comida, considerando sua adequação à idade, sexo, constituição física ou enfermidade ${ }^{24}$.

A predominância do pensamento tecnicista, reforçado pelo discurso baseado em um saber traduzido pela linguagem que configura o jogo da clínica, está intimamente relacionada com a mudança da pergunta "O que é que você tem?" para "Onde lhe dói?". Tal passagem - do ser humano para a doença -, envolve uma geometria dos corpos na qual a verbalização do patológico é eminentemente racional e marcada pela objetividade científica positiva. São as medições instrumentais da quantidade do evento observado que determinam o discurso do profissional de saúde, redistribuindo a relação do significado com o significante e configurando o discurso sobre a doença com base no saber sobre o indivíduo como corpo doente que necessita de intervenção ${ }^{25}$. Isso pode ser fielmente extrapolado para a Nutrição Clínica tal como praticada atualmente, assim como para a pesquisa praticada nesse núcleo de saberes.
Também na pesquisa em Nutrição Clínica, o ser doente não é considerado como um todo, limitando a sua prática aos elementos e segmentos que representam resultados de anormalidade ao serem avaliados através de observações fragmentadas $^{26}$. É importante ter em conta que o corpo não se manifesta apenas através de técnicas objetivas. É necessário considerar a natureza histórica constituída no interior de sua existência social concreta, possibilitando o tratamento através da permanente elaboração e reelaboração no mundo ${ }^{27}$. Os procedimentos e técnicas de avaliação clínica do estado nutricional analisam e avaliam segmentos isolados do corpo, reduzindo ou eliminando a subjetividade humana e as diferenças étnicas e culturais, assim ampliando o espaço das objetividades.

A centralidade da pesquisa clínica nas "doenças biomédicas" e o esquadrinhamento do doente em órgãos ou processos metabólicos se mostram tão mais agudos e intensos, quanto mais especializado o objeto de investigação. Tais saberes biocientíficos não ajudam a construir uma perspectiva mais integral para as pessoas. Pelo contrário, seu saber e as terapêuticas daí derivadas são dirigidos às doenças em seus mecanismos fisiopatogênicos e semiogênicos ou seus respectivos riscos, direcionando-se para o consumo de procedimentos especializados ${ }^{28}$.

Para Caponi29, a saúde não pertence à "ordem dos cálculos, não é o resultado de tabelas comparativas, leis ou médias estatísticas e, portanto, seu estudo não é exclusivo das investigações biomédicas". A interpretação da saúde/ doença não se faz apenas com base em sensações fisiológicas, caracterizadas em termos biológicos ou bioestatísticos, uma vez que a própria leitura dessas sensações é uma construção psicossocial e antropológica. Isso significa que a percepção das sensações enquanto alterações faz parte de um aprendizado que diz respeito a significados socialmente compartilhados ${ }^{30,31}$. Do ponto de vista dos conteúdos, é possível dizer que qualquer tema de saúde é também assunto das Ciências Sociais 
e Humanas, uma vez que todos dizem respeito à vida, ao adoecimento, à morte, a como as pessoas os pensam e os enfrentam e ao que a sociedade e o setor fazem para gerenciá-los ${ }^{32}$.

Como, segundo Luz ${ }^{33}$, as disciplinas tradicionais da área da saúde estão ligadas umbilicalmente à Biologia, seu olhar se dá de modo puramente natural e técnico sobre a vida. Tanto do ponto de vista metodológico como epistemológico, essas disciplinas são incapazes de abarcar a totalidade do fenômeno da vida humana. Por essas razões, as Ciências Humanas e Sociais vêm sendo solicitadas a trabalhar em regime de cooperação interdisciplinar, de forma crescente, no campo da saúde, tendo adquirido relevante papel para instrumentar conceitualmente políticas inovadoras de saúde, que levam em consideração a participação de pacientes e de coletivos de usuários, ao incorporar categorias como as de sujeito, sofrimento e cuidado na atenção à saúde.

Tais perspectivas trazem para o primeiro plano as dimensões subjetivas do adoecimento humano, as quais constituem matéria essencial para a Nutrição Clínica. Trata-se de assumir um movimento de busca de aproximação entre a Alimentação e a Nutrição nos planos conceituais, metodológicos e institucionais, potencializando a geração de conhecimentos e saberes inovadores, com repercussões para a sociedade, em especial no que tange à saúde.

\section{COLABORADORES}

RDA OLIVEIRA e SD PRADO trabalharam em todas as etapas de produção do artigo, desde a concepção até a versão final. MCVS CARVALHO e FR FERREIRA colaboraram na construção do texto e trabalharam na sua versão final.

\section{REFERÊ N CIAS}

1. Prado SD, Gugelmin SA, Mattos RA, Klotz-Silva J, Olivares PS. A pesquisa sobre segurança alimentar e nutricional no Brasil de 2000 a 2005: tendências e desafios. Cienc Saúde Colet. 2010; 15(1):7-18.
2. Klotz-Silva J, Prado SD, Carvalho MCVS, Ornelas TFS, Oliveira PF. Alimentação e cultura como campo científico no Brasil. Physis. 2010; 20(2):413-42.

3. Prado SD, Prado SD, Bosi MLM, Carvalho MCVS, Gugelmin AS, Klotz-Silva J, et al. A pesquisa sobre Alimentação no Brasil: sustentando a autonomia do campo Alimentação e Nutrição. Cienc Saúde Colet. 2011; 16(1):107-19.

4. Prado SD, Bosi MLM, Carvalho MCVS, Gugelmin AS, Mattos RA, Camargo Jr. KR, et al. Alimentação e Nutrição como campo científico autônomo no Brasil: conceitos, domínios e projetos políticos. Rev Nutr. 2011; 24(6):927-38. doi: 10.1590/S1415-52 732011000600013

5. Delmaschio KL. Os grupos de pesquisa nos campos da alimentação e nutrição e das ciências dos alimentos de 2000 a 2008 no Brasil [mestrado]. Rio de Janeiro: Universidade do Estado do Rio de Janeiro; 2012.

6. Bourdieu P. O campo científico. In: Pierre B. Sociologia. São Paulo: Ática; 1983.

7. Bourdieu P. Os usos sociais da ciência: por uma sociologia clínica do campo científico. São Paulo: Unesp; 2004.

8. Khun T. A estrutura das revoluções científicas. São Paulo: Perspectiva; 2003.

9. Chaves N. Nutrição básica e aplicada. Rio de Janeiro: Guanabara Koogan; 1978.

10. Chemin SMSS, Mura JDAP. Tratado de alimentação, nutrição e dietoterapia. São Paulo: Roca; 2007.

11. Oliveira JED, Santos AC, Wilson ED. Nutrição básica. São Paulo: Sarvier; 1982.

12. Philippi ST. Nutrição e técnica dietética. Barueri: Manole; 2003.

13. Coordenação de Aperfeiçoamento de Pessoal de Nível Superior. Comunicado 003/2012 - área Nutrição. Considerações sobre multidisciplinaridade e interdisciplinaridade na área. Brasília: Capes; 2012 [acesso 2012 abril 7] Disponível em: <http//www. capes.gov.br/images/stories/download/avaliação/ interdisciplinaridade_nutricao.pdf $>$.

14. Nunes ED. Saúde coletiva: história recente, passado antigo. In: Campos GWS, Minayo MCS, Akerman M, organizador. Tratado de saúde coletiva. São Paulo: Hucitec; 2009.

15. Kac G, Proença RCP, Prado SD. A criação da área Nutrição na Capes. Rev Nutr. 2011; 24(6):905-16. doi: 10.1590/S1415-52732011000600011

16. Prado SD. Quais os núcleos de saberes que conformam o campo da Alimentação e Nutrição no Brasil? Demetra. 2013; 8(1):1-8.

17. Guimarães R, Lourenço R, Cosac S. A pesquisa em epidemiologia no Brasil. Rev Saúde Pública. 2001; 35(4):321-40. 
18. Conselho Nacional de Desenvolvimento Científico e Tecnológico. Diretório dos grupos de pesquisa no Brasil. Plataforma Lattes. Brasilia: CNPq; 2013 [acesso 2013 maio 15]. Disponível em: <http:// lattes.cnpq.br/web/dgp>.

19. Journal Citation Reports. [cited 2014 May 17]. Available from: <http://thomsonreuters.com/ journal-citation-reports/>.

20. SCImago Journals \& Country Ranks. [cited 2014 May 17]. Available from: <http://www.scimagojr.com/>.

21. Zago MA. A pesquisa clínica no Brasil. Ciênc Saúde Colet. 2004; 9(2):363-74.

22. Zucchetti C, Morrone FB. Perfil da pesquisa clínica no Brasil. Rev HCPA. 2012; 32(3):340-7.

23. Conrad P. The medicalization of society. Baltimore: The Johns Hopkins University Press; 2007.

24. Carneiro HS. Comida e sociedade: significados sociais na história da alimentação. Hist Questões Debates. 2005; 42(0):71-80.

25. Foucault M. O nascimento da clínica. Rio de Janeiro: Forense Universitária; 2006.

26. Canguilhem G. O normal e o patológico. Rio de Janeiro: Forense Universitária; 2002.
27. Silva AM. Corpo, ciência e mercado: reflexões acerca da gestação de um novo arquétipo da felicidade. Campinas: Autores Associados; 2001.

28. Tesser CD, Luz MT. Racionalidades médicas e integralidade. Ciênc Saúde Colet. 2008; 13(1):195-206.

29. Caponi S. A saúde, como abertura ao risco. In: Czeresnia D, organizador. Promoção da saúde: conceitos, reflexões, tendência. Rio de Janeiro: Fiocruz; 2003.

30. Ferreira J. O corpo sígnico. In: Alves PC, Organizador. Saúde e doença: um olhar antropológico. Rio de Janeiro: Fiocruz; 1994.

31. Nordenfelt L. Conversando sobre saúde: um diálogo filosófico. Florianópolis: Bermúncia; 2000.

32. Minayo MCS. A produção de conhecimentos na interface entre as Ciências Sociais e Humanas e a Saúde Coletiva. Saúde Soc. 2013; 22(1):21-31.

33. Luz MT. Especificidade da contribuição dos saberes e práticas das ciências sociais e humanas para a saúde. Saúde Soc. 2011; 20(1):22-31.

Recebido em: 16/4/2014 Aprovado em: 6/11/2014 